\title{
Controle de Alternaria solani em Tomateiro por Extratos de Curcuma longa e Curcumina - II. Avaliação in vivo
}

\author{
María I. Balbi-Peña ${ }^{1}$, Andrea Becker ${ }^{1}$, José Renato Stangarlin', Gilmar Franzener ${ }^{1}$, \\ Mário C. Lopes ${ }^{1} \&$ Kátia R. F. Schwan-Estrada ${ }^{2}$ \\ ${ }^{1}$ Centro de Ciências Agrárias, Universidade Estadual do Oeste de Paraná, Unioeste, Campus de Marechal Cândido Rondon, \\ Cx. Postal 1008, CEP 85960-000, Marechal Cândido Rondon, PR, e-mail: jrstangarlin@unioeste.br; ${ }^{2}$ Departamento de \\ Agronomia, Universidade Estadual de Maringá, UEM, CEP 87020-900, Maringá, PR
}

(Aceito para publicação em 01/06/2006)

Autor para correspondência: José Renato Stangarlin

BALBI-PEÑA, M.I., BECKER, A., STANGARLIN, J.R., FRANZENER, G., LOPES, M.C. \& SCHWAN-ESTRADA, K.R.F. Controle de Alternaria solani em tomateiro por extratos de Curcuma longa e curcumina - II. Avaliação in vivo. Fitopatologia Brasileira 31:401-404. 2006.

\section{RESUMO}

A pinta preta, causada por Alternaria solani, é uma das mais importantes doenças da cultura do tomateiro no Brasil. Várias alternativas aos fungicidas têm sido avaliadas nos últimos anos na busca de produtos que controlem satisfatoriamente as doenças, tenham pequeno impacto ambiental e baixa toxicidade aos seres vivos. A cúrcuma, Curcuma longa, apresenta em seus rizomas compostos com comprovada atividade antimicrobiana. Dessa forma, o objetivo deste trabalho foi avaliar o controle de pinta preta em tomateiro utilizando extratos de cúrcuma e curcumina em condições de casa de vegetação. Os tratamentos utilizados foram: extrato de cúrcuma (1 e 10\%), curcumina (50 e $100 \mathrm{mg} / \mathrm{L})$, acibenzolar-S-metil (ASM) (25 $\mathrm{mg}$ do i.a./L), oxicloreto de cobre (1.100 $\mathrm{mg}$ do i.a./L), azoxystrobin ( $80 \mathrm{mg}$ do i.a./L) e testemunha (água). A curcumina e os extratos brutos de cúrcuma apresentaram níveis de controle de pinta preta similares ao tratamento com fungicida cúprico, mas inferior ao azoxystrobin. Não houve diferenças estatísticas na produção comercial de tomate entre tratamentos. Somente o tratamento de curcumina $50 \mathrm{mg} / 1$ apresentou maior porcentagem de frutos grandes em relação à testemunha. Esses resultados indicam o potencial de controle de pinta preta em tomateiro com cúrcuma e curcumina.

Palavras-chave adicionais: cúrcuma, extratos de plantas, pinta preta, controle alternativo de doenças de plantas.

\begin{abstract}
Control of Alternaria solani in tomato by Curcuma longa extracts and curcumin - II. In vivo evaluation.

Early blight, caused by Alternaria solani, is one of the most important diseases of tomato in Brazil. New alternatives to fungicides with low environmental impact and low toxicity to living beings have been tested to evaluate their ability to control diseases. Turmeric, Curcuma longa, has compounds in its rhizomes with proven antimicrobial activity. Therefore, the objective of this study was to evaluate the potential of turmeric extracts and curcumin on the control of tomato early blight under greenhouse conditions. The treatments were: 1 and $10 \%$ turmeric extracts, 50 and $100 \mathrm{mg} / \mathrm{L}$ curcumin solutions, acibenzolar-S-metil (ASM) (25 mg a.i./L), copper oxychloride (1,100 $\mathrm{mg}$ a.i./L), azoxystrobin $(80 \mathrm{mg}$ a.i./L) and control (water). The disease control showed by turmeric extracts and curcumin solutions was similar to cupric fungicide, but inferior to azoxystrobin control. There was no statistical difference in commercial fruit production between treatments. Only 50 $\mathrm{mg} / \mathrm{L}$ curcumin treatment had a higher percentage of bigger fruits compared to the control. These results show the potential of turmeric and curcumin for the control of tomato early blight.
\end{abstract}

Additional keywords: turmeric, plant extracts, tomato early blight, alternative plant disease control.

A pinta preta, causada por Alternaria solani (Ellis \& G. Martin) L.R. Jones \& Grout, é uma das mais importantes e freqüentes doenças da cultura do tomateiro no Brasil. A doença apresenta alto potencial destrutivo, incidindo sobre folhas, hastes, pecíolos e frutos, ocasionando elevados prejuízos econômicos. Atualmente, o pequeno número de cultivares com resistência genética a essa doença, associado ao alto custo de suas sementes, determinam medidas de controle basicamente com produtos químicos para as variedades tradicionalmente cultivadas, que são suscetíveis ao patógeno (Vale et al., 2000).
Trabalhos desenvolvidos com extrato bruto ou óleo essencial, obtidos a partir de plantas medicinais, têm indicado o potencial das mesmas no controle de fitopatógenos, tanto por sua ação fungitóxica direta quanto pela indução de resistência, indicando a presença de compostos com características de eliciadores. Como exemplos tem-se o controle da mancha marrom [Bipolaris sorokiniana (Sacc.) Shoemaker] em trigo usando extrato aquoso de Artemisia camphorata Vill. (cânfora) (Franzener et al., 2003), do oídio (Oidium lycopersici Cooke \& Massee) do tomateiro pelo óleo 
emulsionável de Azadirachta indica A. Juss (Carneiro, 2003), da antracnose [Colletotrichum lagenarium (Pass.) Ellis \& Halst.] em pepino pelo extrato de Eucalyptus citriodora Hooker (Bonaldo et al., 2004) e do mofo branco (Sclerotinia sclerotiorum (Lib.) de Bary) em alface por Zingiber officinale Roscoe (Rodrigues, 2004).

No caso da cúrcuma, Curcuma longa L., os trabalhos encontrados na literatura referem-se basicamente ao estudo da atividade antimicrobiana in vitro de seus compostos, como contra Colletotrichum gloeosporioides (Penz.) Penz. \& Sacc., Sphaceloma cardamomi Muthappa, Pestalotia palmarum Cooke, Rhizoctonia solani J.G. Kühn, Aspergillus sp. e Fusarium sp. (Saju et al., 1998), Colletotrichum falcatum Went, Fusarium moniliforme J. Sheld., Curvularia pallescens Boedijn, Aspergillus niger Thiegh. e Fusarium oxysporum Schlecht. (Singh et al., 2002).

Há apenas dois trabalhos verificando o potencial de cúrcuma no controle de doenças. Em um estudo sobre o efeito de C. longa em Xanthomonas axonopodis pv. manihotis (Bondar) Vauterin, Hoste, Kersters \& Swings., o extrato apresentou ação bactericida in vitro para concentrações que variaram de 10 a $20 \%$, dependendo da procedência dos rizomas. No entanto, in vivo, nas concentrações utilizadas, não houve efeito curativo em manivas de mandioca infectadas com o patógeno (Kuhn et al., 2006). O tratamento com curcumina a 50 $\mathrm{mg} / \mathrm{L}$ proporcionou um peso total de sementes de soja $29 \%$ maior que o obtido com o tratamento padrão com o fungicida pyraclostrobin + epoxiconazole, em ensaio para controle de doenças de final de ciclo, como Septoria glycines Hemmi, Cercospora kikuchi (Tak. Matsumoto \& Tomoy.) M.W. Gardner e oídio (Microsphaera diffusa Cooke \& Peck) em condições de campo (Becker et al. 2004).

Com relação ao tomateiro, não há nenhum relato sobre o uso de cúrcuma para controle de $A$. solani. Dessa forma, o objetivo deste trabalho foi avaliar o efeito da aplicação de extrato aquoso de $C$. longa e curcumina no controle da pinta preta do tomateiro cultivado em ambiente protegido.

Foram utilizados isolados obtidos a partir de lesões de plantas de tomateiro infectadas naturalmente $\operatorname{com} A$. solani, coletadas no município de Marechal Cândido Rondon PR. Para manutenção e produção de esporos, o fungo foi cultivado em meio de cultura batata-dextroseágar (BDA) e incubado a $25^{\circ} \mathrm{C}$ e fotoperíodo de $12 \mathrm{~h}$.

Sementes de tomateiro da cultivar Bônus F1 foram semeadas em bandejas de isopor de 128 células contendo substrato Plantmax $\mathrm{HA}^{\circledR}$. As mudas foram transplantadas aos 28 dias após a semeadura (DAS) para canteiros em casa de vegetação. $O$ espaçamento adotado foi $1,2 \mathrm{~m}$ entre linhas e $0,6 \mathrm{~m}$ entre plantas na linha. Utilizaramse as quatro linhas centrais para o ensaio, deixando-se duas linhas de bordadura. A acidez do solo foi corrigida através de calcário $(1500 \mathrm{~kg} / \mathrm{ha})$ e a adubação de base foi feita com $3000 \mathrm{~kg} / \mathrm{ha}$ da fórmula 4-14-8 (NPK) mais micronutrientes. Após o transplante, iniciou-se a fertirrigação por gotejamento a cada três dias, sendo a quantidade de água estimada em $1 \mathrm{~L} /$ planta/dia. Foram utilizados os fertilizantes solúveis Kristalon da Utilfértil Fertilizantes (Itapetininga/SP), segundo as seguintes dosagens: de 15 a 45 dias após transplante (DAT), Kristalon branco (15-5-30) na dosagem de 0,6 $\mathrm{g} / \mathrm{L} / \mathrm{dia}$, nitrato de cálcio $0,3 \mathrm{~g} / \mathrm{L} /$ dia e 'Hydro cocktail' $4 \mathrm{~g} / \mathrm{L} / \mathrm{dia}$; de 45 a 75 DAT, Kristalon laranja (6-12-36) na dosagem de 0,4 g/L/ dia, nitrato de cálcio $0,7 \mathrm{~g} / \mathrm{L} /$ dia e 'Hydro cocktail' $5 \mathrm{~g} /$ L/dia; de 75 DAT até o final do cultivo, Kristalon laranja (6-12-36) na dosagem de $1 \mathrm{~g} / \mathrm{L} /$ dia, nitrato de cálcio 0,3 $\mathrm{g} / \mathrm{L} /$ dia e 'Hydro cocktail' 5,8 g/L/dia.

O delineamento experimental usado foi o de blocos ao acaso com quatro repetições, sendo cada parcela composta por cinco plantas. Foram feitas seis aplicações dos produtos referentes a cada tratamento aos $33,50,65,83,102$ e 123 DAT. Os tratamentos utilizados foram: extrato de cúrcuma (1 e 10\%), curcumina (50 e $100 \mathrm{mg} / \mathrm{L})$, acibenzolar-S-metil (ASM) (25 mg de i.a./L), oxicloreto de cobre (1.100 $\mathrm{mg}$ de i.a./L), azoxystrobin (80 mg de i.a./L) e testemunha (água). Os rizomas de cúrcuma foram coletados na região de Mercedes PR e armazenados a $-20{ }^{\circ} \mathrm{C}$. Foram triturados em liquidificador com água destilada, na dosagem de 10 g e 100 g por litro, constituindo extratos líquidos a 1 e $10 \%$, respectivamente. As aplicações foram realizadas com pulverizador manual até plena cobertura foliar. As plantas foram inoculadas duas vezes. A primeira inoculação foi feita aos 36 DAT na concentração de $1 \times 10^{4}$ conídios $/ \mathrm{mL}$. Realizou-se uma segunda inoculação aos 53 DAT na concentração de 3,6 x $10^{4}$ conídios $/ \mathrm{mL}$ devido à ausência de sintomas até essa data. Nas duas inoculações foi adicionado o espalhante Tween-20 (uma gota por cada $300 \mathrm{~mL}$ de suspensão).

As variáveis avaliadas neste ensaio foram:

a) Severidade da doença nas folhas. A pinta preta foi avaliada através de adaptação da escala diagramática de Boff (1988), em que se atribuíram notas: 1 - ausência de sintomas; 2 - de traços de sintomas a $4 \%$ de severidade; 3 - 4,1 a $8 \% ; 4-8,1$ a $16 \% ; 5-16,1$ a $32 \% ; 6$ - acima de $32 \%$ de área foliar lesionada. Foram realizadas sete avaliações $(68,77,83,91,105,131$ e 152 DAT) adotando-se como padrão a escolha ao acaso de cinco folíolos por planta, pertencentes aos terços médio e inferior das três plantas centrais da parcela. A primeira avaliação foi realizada quando as plantas controle apresentaram sintomas.

Com as avaliações de severidade de pinta preta nas folhas, obteve-se as curvas de progresso da pinta preta. A partir das curvas, calculou-se a área abaixo da curva de progresso da pinta preta (AACPPP) conforme equação proposta por Shaner \& Finney (1977). Com os dados de AACPPP foi feita a análise de variância e aplicou-se o teste de Tukey a $5 \%$ de probabilidade para comparação das médias. 
b) Número de frutos sadios, massa fresca de frutos, classificação de frutos e produção comercial. Foram avaliados através de contagens e pesagens de todos os frutos colhidos das três plantas centrais das diferentes colheitas. Para o cálculo de produção comercial foram considerados somente aqueles frutos com diâmetro maior que $40 \mathrm{~mm}$ e calculada a produção por planta. Para a análise estatística tomaram-se os dados totais das diferentes colheitas, sendo as médias comparadas pelo teste de Tukey a $5 \%$ de probabilidade.

A classificação de frutos por tamanho foi avaliada utilizando-se uma adaptação da proposta pelo Programa Brasileiro para Melhoria dos Padrões Comerciais e Embalagens de Hortigranjeiros, em que foram considerados: frutos pequenos - diâmetro de 40 a 50 $\mathrm{mm}$; frutos médios - de 51 a $60 \mathrm{~mm}$; e frutos grandes - acima de $61 \mathrm{~mm}$. Posteriormente, foram calculadas as porcentagens de frutos por tamanho e, antes da análise estatística, transformados em arc sen da raiz de $\mathrm{x} / 100$.

A pinta preta apresentou elevada severidade, determinada pelas duas inoculações realizadas com esporos do patógeno e favorecida pela suscetibilidade da cultivar e pela ocorrência de condições ambientais favoráveis à doença (dados climáticos não apresentados).

Foi calculada a área abaixo da curva de progresso da pinta preta (AACPPP) para comparar os tratamentos (Figura 1). Todos os tratamentos apresentaram valor de AACPPP estatisticamente menor do que a testemunha. Dentre os mesmos, o tratamento com ASM foi o menos eficiente, mas não diferindo estatisticamente de cúrcuma $10 \%$ e curcumina $100 \mathrm{mg} / \mathrm{L}$. Cúrcuma a $1 \%$, curcumina $50 \mathrm{mg} / \mathrm{L}$ e oxicloreto de cobre apresentaram melhor nível

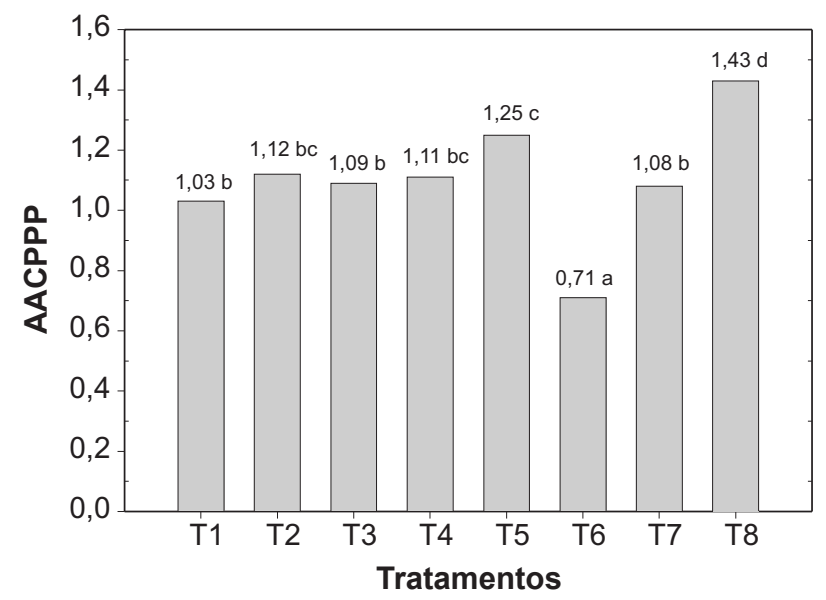

FIG. 1 - Área abaixo da curva de progresso da pinta preta (AACPPP) em plantas de tomateiro mantidas em cultivo protegido com oito tratamentos: cúrcuma 1\% (T1), cúrcuma 10\% (T2), curcumina 50mg/l (T3), curcumina $100 \mathrm{mg} / \mathrm{l}$ (T4), Acibenzolar-Smetil (ASM) (T5), Azoxystrobin (T6), Oxicloreto de cobre (T7) e testemunha inoculada (T8). Letras distintas representam diferenças significativas ao nível de $5 \%$ pelo teste de Tukey. de controle, mas ainda inferior estatisticamente ao obtido pelo fungicida azoxystrobin. $\mathrm{O}$ indutor de resistência ASM tem apresentado eficiências distintas conforme o patossistema envolvido. Em trabalhos realizados com ASM contra Xanthomonas campestris pv. vesicatoria (Doidge) Dye et al., Oidium lycopersici e Septoria lycopersici Speg. em tomateiro, houve proteção contra os dois primeiros patógenos mas não contra $S$. lycopersici (Silva, 2002).

Em nenhum momento foram observados sintomas de fitotoxidez nos tomateiros tratados com EB de cúrcuma e solução de curcumina, diferentemente do que se observou com o patossistema mandioca $-X$. axonopodis pv. manihotis, no qual o tratamento de manivas com este extrato causou redução de brotamento (Kuhn et al., 2006).

As diferenças observadas nos valores de severidade nas folhas podem ter ocorrido por atividade antimicrobiana direta dos extratos de cúrcuma e curcumina, já que in vitro tais tratamentos foram capazes de inibir em até $15 \%$ a germinação de esporos e em até $38 \%$ o crescimento micelial de A. solani (Balbi-Peña, 2005). Entretanto, não se pode descartar a possibilidade de também estar ocorrendo indução de resistência nessas plantas de tomateiro, fato já constatado em outros patossistemas utilizando extratos de outras plantas medicinais (Franzener et al., 2003; Bonaldo et al., 2004). De qualquer forma, os resultados aqui obtidos podem ser enquadrados no conceito de controle alternativo de doenças, o qual envolve o uso de produtos com atividade antimicrobiana direta e/ou indutora de resistência (Schwan-Estrada et al., 2003).

$\mathrm{Na}$ literatura foi encontrado apenas um relato sobre o controle de A. solani em tomateiro por extratos vegetais. Montes-Belmont \& Garcia-Licona (1997), verificando a eficiência da infusão de folhas secas de 50 espécies de plantas no controle de pinta preta, observaram que todos os extratos reduziram o número de lesões por planta durante o primeiro estágio de desenvolvimento da doença e que, posteriormente, somente os extratos de Allium sativum L., Teloxys ambrosioides (L.) W.A. Weber e Tagetes erecta L. reduziram a severidade em relação ao tratamento com maneb. A produção do tomateiro não foi avaliada por esses pesquisadores.

Referente aos frutos, não houve diferenças estatísticas na produção e no número de frutos comerciais, nem na massa fresca média de frutos entre tratamentos (Tabela 1), bem como não foram constatados frutos doentes com sintomas de pinta preta. Quanto ao tamanho dos frutos, o tratamento de curcumina $50 \mathrm{mg} / \mathrm{L}$ foi o único que apresentou menor porcentagem de frutos pequenos e maior de frutos grandes em relação à testemunha. Não houve diferença significativa entre os tratamentos com relação à porcentagem de frutos médios.

A utilização de extrato bruto de cúrcuma ou curcumina pode ser uma opção para controle de pinta preta em cultivos orgânicos de tomate, já que resulta em 
TABELA 1 - Produção comercial de tomateiro mantido em cultivo protegido e porcentagem de frutos comerciais em plantas inoculadas com $A$. solani e tratadas com diferentes produtos. Frutos pequenos: diâmetro de 41 a $50 \mathrm{~mm}$, médios: diâmetro de 51 a 60 mm e grandes: diâmetro $>61 \mathrm{~mm}$

\begin{tabular}{|c|c|c|c|c|}
\hline \multirow{2}{*}{ TRATAMENTOS } & \multirow{2}{*}{$\begin{array}{c}\text { Produção comercial } \\
\text { (kg/planta) }\end{array}$} & \multicolumn{3}{|c|}{ Tamanho de frutos $(\%)$} \\
\hline & & Pequenos & Médios & Grandes \\
\hline Cúrcuma 1\% & $6,56 \mathrm{a}$ & $19,30 a b^{1}$ & $44,28 \mathrm{a}$ & $36,42 \mathrm{ab}$ \\
\hline Cúrcuma $10 \%$ & $5,80 \mathrm{a}$ & $21,75 a b$ & $45,74 \mathrm{a}$ & $32,51 \mathrm{ab}$ \\
\hline Curcumina $50 \mathrm{mg} / \mathrm{l}$ & $6,72 \mathrm{a}$ & $17,15 \mathrm{~b}$ & 44,53 a & $38,32 \mathrm{a}$ \\
\hline Curcumina 100 mg/l & $6,06 \mathrm{a}$ & $20,37 \mathrm{ab}$ & $45,32 \mathrm{a}$ & $34,32 \mathrm{ab}$ \\
\hline $\mathrm{ASM}^{2}$ & $5,89 \mathrm{a}$ & $24,27 \mathrm{ab}$ & $52,94 \mathrm{a}$ & $22,79 \mathrm{ab}$ \\
\hline Azoxystrobin & $6,14 \mathrm{a}$ & $29,79 a b$ & $41,36 \mathrm{a}$ & $28,85 \mathrm{ab}$ \\
\hline Oxicloreto de $\mathrm{Cu}$ & $6,65 \mathrm{a}$ & $27,42 \mathrm{ab}$ & $46,57 \mathrm{a}$ & $26,01 \mathrm{ab}$ \\
\hline Testemunha & $5,90 \mathrm{a}$ & $31,71 \mathrm{a}$ & $47,20 \mathrm{a}$ & $21,09 \mathrm{~b}$ \\
\hline CV $(\%)$ & 10,5 & 13,5 & 10,5 & 13,3 \\
\hline
\end{tabular}

${ }^{1}$ Médias na coluna, seguidas de mesma letra, não diferem entre si pelo teste de Tukey ao nível de 5\% de probabilidade. Médias originais. Para análise estatística, os dados foram transformados em arc sen da raiz quadrada de $(\mathrm{x} / 100)$

${ }^{2}$ Acibenzolar-S-metil.

níveis de severidade e produtividade similares aos obtidos com fungicidas cúpricos.

\section{AGRADECIMENTOS}

A primeira autora agradece à concessão de bolsa pela CAPES; JRS e KRFS à concessão de bolsa pelo CNPq.

\section{REFERÊNCIAS BIBLIOGRÁFICAS}

BALBI-PEÑA, M.I. Efeito do extrato do rizoma de Curcuma longa e solução de curcumina em Alternaria solani e controle de pinta preta em tomateiro. Dissertação de Mestrado. Marechal Cândido Rondon PR. Universidade Estadual do Oeste de Paraná. 2005.

BECKER, A., VIGO-SCHULTZ, S.C., STANGARLIN, J.R., BALBI-PEÑA, M.I., KLAHOLD, C.A. \& SCHWAN-ESTRADA, K.R.F. Controle alternativo das doenças de final de ciclo e oídio na cultura da soja. Fitopatologia Brasileira 29(Supl.):163. 2004. (Resumo)

BOFF, P. Epidemiologia e controle químico da mancha de estenfílio (Stemphylium solani Weber) e da pinta preta (Alternaria solani (Ellis \& Martin) Jones \& Grout) em dois sistemas de condução do tomateiro (Lycopersicon esculentum Mill.). Dissertação de
Mestrado. Viçosa MG. Universidade Federal de Viçosa. 1988. BONALDO, S.M., SCHWAN-ESTRADA, K.R.F., STANGARLIN, J.R., TESSMANN, D.J. \& SCAPIM, C.A. Fungitoxicidade, atividade elicitora de fitoalexinas e proteção de pepino contra Colletotrichum lagenarium pelo extrato aquoso de Eucalyptus citriodora. Fitopatologia Brasileira 29:128-134. 2004.

CARNEIRO, S.M.T.P.G. Efeito de extratos de folhas e do óleo de nim sobre o oídio do tomateiro. Summa Phytopathologica 29:262265. 2003.

FRANZENER, G., STANGARLIN, J.R., SCHWAN-ESTRADA, K.R.F. \& CRUZ, M.E.S. Atividade antifúngica e indução de resistência em trigo a Bipolaris sorokiniana por Artemisia camphorata. Acta Scientiarum 25:503-507. 2003.

KUHN, O.J., PORTZ, R.L., STANGARLIN, J.R., DEL ÁGUILA, R.M., SCHWAN-ESTRADA, K.R.F. \& FRANZENER, G. Efeito do extrato aquoso de cúrcuma (Curcuma longa) em Xanthomonas axonopodis pv. manihotis. Semina Ciências Agrárias 27:13-20. 2006.

MONTES-BELMONT, R. \& GARCIA-LICONA, R. Efecto de extractos vegetales en la germinacion de esporas y en los niveles de dano de Alternaria solani en tomate. Fitopatologia (Peru) 32:5257. 1997.

RODRIGUES, E. Atividade antimicrobiana in vitro, indução de peroxidase e controle de Sclerotinia sclerotiorum em alface cultivado organicamente pelo uso de extrato de gengibre. Dissertação de Mestrado. Maringá PR. Universidade Estadual de Maringá. 2004.

SAJU, K.A., VENUGOPAL, M.N. \& MATHEW, M.J. Antifungal and insect-repellent activities of essential oil of turmeric (Curcuma longa L.). Current Science 75:660-662. 1998.

SCHWAN-ESTRADA, K.R.F., STANGARLIN, J.R. \& CRUZ, M.E.S. Uso de plantas medicinais no controle de doenças de plantas. Fitopatologia Brasileira 28:554-556. 2003.

SHANER, G. \& FINNEY, R.E. The effect of nitrogen fertilizationon the expression of slow mildewing resistance in Knox wheat. Phytopathology 67:1051-1056. 1977.

SILVA, L.H.C.P. Resistência sistêmica ativada pelo acibenzolarS-metil contra doenças em tomateiro. Dissertação de Mestrado. Lavras MG. Universidade Federal de Lavras. 2002.

SINGH, G., SINGH, O.P. \& MAURYA, S. Chemical and biocidal investigations on essential oils of some Indian Curcuma species. Progress in Crystal Growth and Characterization of Materials 45:75-81. 2002.

VALE, F.X.R., ZAMBOLIM, L., PAUL, P.A. \& COSTA, H. Doenças causadas por fungos em tomate. In: Zambolim, L., Vale, F.X.R. \& Costa, H. (Eds.) Controle de Doenças de Plantas: Hortaliças. Viçosa MG. Universidade Federal de Viçosa. 2000. pp. 699-756. 